\title{
Image Registration of Low-Signal-to-Noise STEM Data with Open Source Software
}

Benjamin H. Savitzky ${ }^{1,5}$, Ismail El Baggari ${ }^{1}$, Colin B. Clement ${ }^{1}$, Emily Waite ${ }^{2}$, Robert Hovden ${ }^{2,4}$ and Lena F. Kourkoutis ${ }^{2,3}$

1. Department of Physics, Cornell University, Ithaca, NY, USA.

2. School of Applied \& Engineering Physics, Cornell University, Ithaca, NY, USA.

3. Kavli Institute for Nanoscale Science, Cornell University, Ithaca, NY, USA.

4. Present address: Dept. of Mater. Sci. and Eng., University of Michigan, Ann Arbor, MI, USA.

5. Present address: NCEM, Molecular Foundry, LBNL, Berkeley, CA, USA.

* Corresponding authors: bhsavitzky@lbl.gov \& lena.f.kourkoutis@cornell.edu

Aligning and averaging stacks of fast-acquisition STEM images to improve signal-to-noise ratios (SNRs) has become an increasingly important tool for high precision, quantitative analysis of STEM data [1, 2, 3]. However, low SNRs in the raw images can make image registration untenable with standard approaches. Moreover, in atomic resolution imaging, high translational symmetry in crystalline samples can introduce pernicious "unit cell hops" in image alignments, resulting in subtly specious averaged images. Particularly in light of the possibility of such subtle errors, source code availability is crucial to ensure reproducibility and fidelity of results. Here, we present a free and open source python package implementing a new approach to image registration which enables alignment of STEM image stacks even in the presence of low SNRs and high translational symmetry [4].

Cryogenic imaging of a manganite crystal provides a case study (Fig. 1). While cryo-STEM enables characterization of previously inaccessible materials' phases, it also introduces additional stage instability due to the cooling process. This can be partially mitigated by fast image acquisitions, however, the SNR of the resulting data is generally quite low (Fig. 1a-c). Using our image registration approach, we achieve high-SNR average images information transfer as low as $0.6 \AA$ at $93 \mathrm{~K}$ (Fig. 1d-f).

Our approach does not rely on a single reference image, but instead uses all possible combinations of image correlations to determine the optimal shifts [4,5]. This results in an information surplus, enabling enforcement of physical consistency between all measured relative image shifts. Consequently, the incorrect correlations which plague low-SNR data may be identified and corrected (Fig. 2). Moreover, the criterion of physical consistency may be used to substantiate the validity of the registration.

Lattice images constitute a common and important class of images requiring special care, as highly translationally symmetric data has a propensity to register incorrect unit-cell jumps. Tools to avoid or account for these and other errors will be discussed, including judicious Fourier space weighting, or preemptive avoidance of sampling errors (Fig. 3). In combination with the physical consistency constraints, we demonstrate how to verify that the final image registration is free of these errors.

References:

[1] X Sang and JM LeBeau, Ultramicroscopy 138 (2014), p. 28.

[2] B Berkels et al., Ultramicroscopy 138 (2014), p. 46.

[3] C Ophus, J Ciston and CT Nelson, Ultramicroscopy. 162 (2016), p. 1.

[4] BH Savitzky et al., Ultramicroscopy, 191 (2018), p. 56.

[5] X Li et al., Nature Methods, 10 (2013), p. 584. 
[6] BHS acknowledges support from the NSF GRFP (DGE-1144153). This work was supported by NSF (DMR-1539918, DMR-1429155, DMR-1719875) and DOD AFOSR (FA 9550-16-1-0305). All associated code is open source and freely available at https://github.com/bsavitzky/rigidRegistration, and can be conveniently run through the iPython/Jupyter notebook.

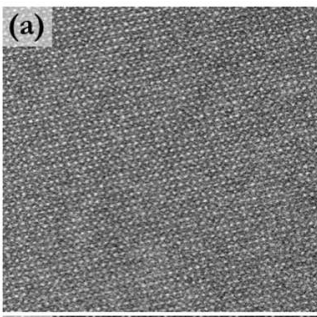

(d)

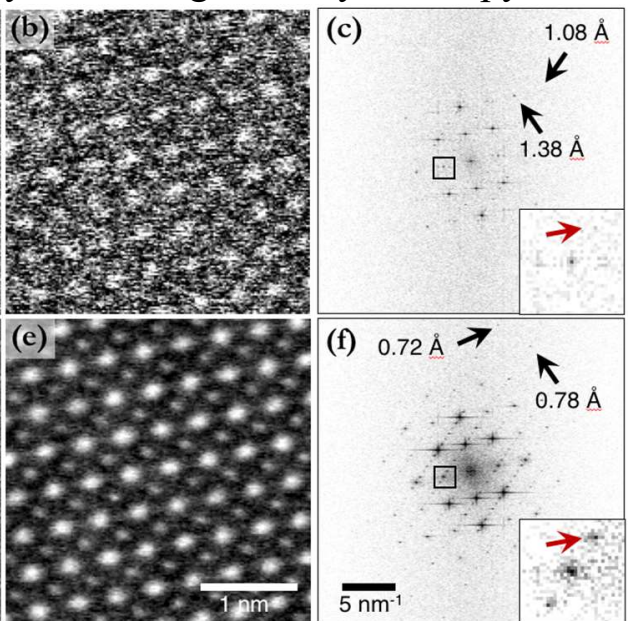

Figure 1. Registration of low SNR cryo-STEM data. (a-c) A single fast acquisition (0.63 s) frame from a 40 image series of a $\mathrm{Bi}_{1-\mathrm{x}} \mathrm{Sr}_{\mathrm{x}-}$ $\mathrm{y}_{\mathrm{y}} \mathrm{MnO}_{3}$ (BSCMO) crystal imaged under liquid nitrogen cooling. In the full field of view (a) and zoom-in (b) the brighter A-sites of the perovskite lattice are identifiable but noisy, while the dimmer B-sites cannot be clearly distinguished. The FFT (c) shows clear information transfer to $1.38 \AA$, and very weak reflections at $1.08 \AA$. (d-f) Registering and averaging all 40 images by determining the optimal shifts from all image pairs yields a high SNR final image (a), clearly identifiable A- and B-sites (b), and information transfer to $0.72 \AA$ (c). BSCMO hosts a charge density wave state which breaks translational symmetry, manifesting as satellite peaks in the FFT (red arrows), which are undetectable in a single frame but are immediately apparent in the registered data.

Figure 2. Referenceless cryo-STEM (a) image correlation. (a) All possible image pairs in a stack of fast acquisition cryo-STEM images are cross correlated to determine their relative shifts. (b) The shift matrices $R_{i j}=X_{i j}+Y_{i j} \quad$ visualize the calculated shifts between all image pairs $(i, j)$ (left), from which the optimum global image shifts may be

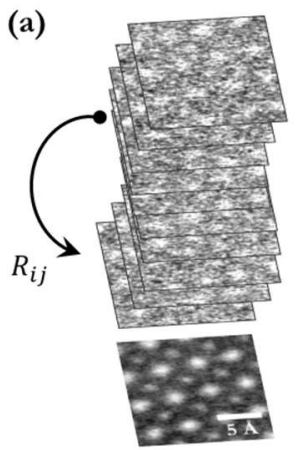

(b)

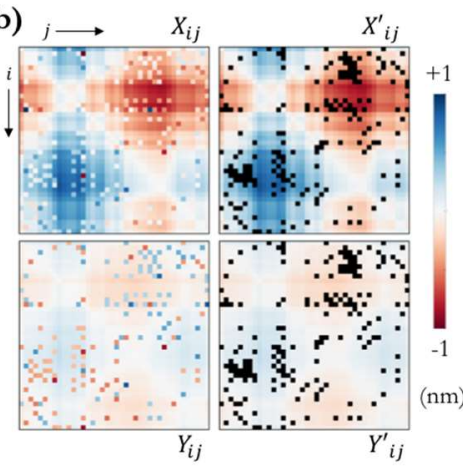

(c)

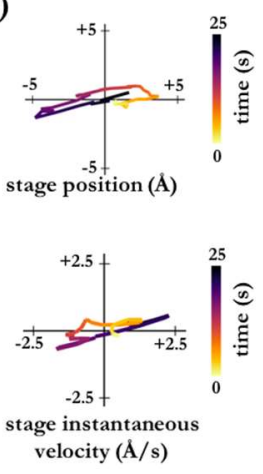

calculated. The smoothly varying background encodes stage movement during acquisition (c), while the aberrant pixels are incorrect correlations, resulting from the low-SNR of the images. False correlations can thus be identified (b, right) and corrected.

Figure 3. Unit cell jumps and sampling error. (a) The cross correlation between a pair of atomic resolution cryo-STEM images contains many local maxima, corresponding to crystal lattice vector offsets between the images. (b) In this case, the correct maximum is highlighted by the black box, however, the brightest pixel is in the local maximum in the red box, due to sampling effects. Extra care is therefore required to avoid "unit cell hopping" during registration. (c) Here, Gaussian fits to multiple local maxima in the cross correlation reduces erroneous shift matrix elements by $50 \%$.
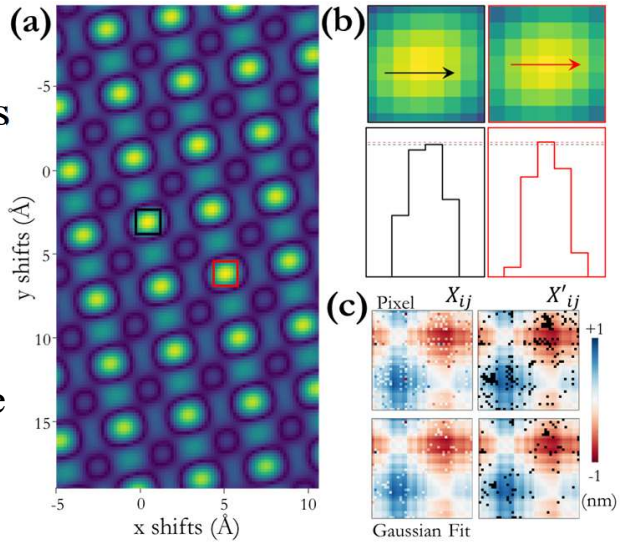